\title{
Older adults using multi-dose dispensing exposed to risks of potentially inappropriate medications
}

\author{
Veera Bobrova $^{\mathrm{a}, *}$, Jyrki Heinämäki $^{\mathrm{a}}$, Outi Honkanen ${ }^{\mathrm{b}}$, Shane Desselle ${ }^{\mathrm{c}}$, Marja Airaksinen ${ }^{\mathrm{d}}$, \\ Daisy Volmer ${ }^{\mathrm{a}}$ \\ ${ }^{a}$ Institute of Pharmacy, Faculty of Medicine, University of Tartu, Estonia \\ ${ }^{\mathrm{b}}$ PharmaService Ltd., Finland \\ ${ }^{\mathrm{c}}$ Touro University California College of Pharmacy, Vallejo, CA, USA \\ ${ }^{\mathrm{d}}$ Division of Pharmacology and Pharmacotherapy, Faculty of Pharmacy, University of Helsinki, Finland
}

\section{A R T I C L E I N F O}

\section{Keywords:}

Estonia

Drug-related problems

Medication review

Medication safety

Multi-dose dispensing

PIMs

\begin{abstract}
A B S T R A C T
Background: Multi-dose dispensing (MDD) of medications is a health technology designed to promote medication adherence and patient safety. MDD has been used as an alternative to ordinary prescription dispensing for patients, mostly elderly with high medication use.

Objective: To evaluate the initiation phase of the MDD service to older adults $\geq 65$ years and assess wheter the medication use of the new MDD patients is appropriate in terms of drug related problems.

Methods: The European Union EU(7)-PIM list and the Inxbase databases were used for identifying potentially inappropriate medications (PIMs) and drug-drug interactions (DDIs). The study sample consisted of a total of 208 patients aged 65-108 years who were involved in the MDD service (PharmaService Ltd.) in Finland in 2015-2016. Clinically significant differences of PIM and DDI occurrences were identified using a Pearson's chisquare test throughout the demographic groups under study.

Results: Results demonstrate that for $81 \%$ of the study participants, at least one medication from the EU (7)-PIM list was prescribed, and up to $64 \%$ of PIMs were clinically significant. According to the Inxbase database, five patients (2.4\%) were prescribed category D clinically significant DDIs. Additionally, $61 \%$ of the patients saw an increase in the number of medications prescribed within six months after the initial MDD order.

Conclusions: The results suggest that the MDD service should be accompanied by a regular medication review tailored to specific patient groups (i.e., older patients) to avoid potential DRPs.
\end{abstract}

\section{Introduction}

Multi-dose dispensing (MDD) is a health technology designed to promote medication adherence and patient safety and it is widely used particularly in the Nordic countries. ${ }^{1-3}$ With MDD, the regularly used medicines, usually in per oral formulation, are machine-packed into unit-dose pouches labelled with the patient's data, medication contents, and timing of dose intake. ${ }^{2}$ The service is most applicable for older patients with multiple medications, those with a limited ability to maintain appropriate medication use, and whose medication regimen is not frequently changed. ${ }^{3}$

There is quite limited evidence on the outcomes of the MDD on appropriate medication use in older adults. ${ }^{1}$ MDD service has been shown to reduce dispensing errors, increase adherence, decrease medication costs, and reduce medication administration time for nurses. ${ }^{3,4}$
The MDD procedure has found to need more standardization, particularly medication reconciliation and review for MDD patients. ${ }^{1}$ The MDD procedure has been recommended to include medication reconciliation and review to all patients prior their enrolment in the service. Action should be taken to ensure that the medication list is clinically appropriate and matches patient's prescribed medication regimen. ${ }^{1,2}$ As minimum, the dosages, administration times of the medications, drugdrug interactions (DDIs) and potentially inappropriate medication (PIM) use should be reviewed by the prescriber and the team responsible for the care of the patient. ${ }^{1}$ However, few studies have been conducted to show the risk of using potentially inappropriate medications (PIMs) by patients on MDD service. ${ }^{4-6}$ This study will focus on the initiation phase of the MDD service to older adults $\geq 65$ years and assess whether the medication of the new MDD service users is appropriate in terms of PIMs and clinically significant DDIs.

\footnotetext{
* Corresponding author.

E-mail address: veera.bobrova@ut.ee (V. Bobrova).
} 


\section{Methods}

\section{Study design and method}

A retrospective registry-based study was carried out in Finland where MDD service was first launched in 2002 and implemented through legislation in $2011 .^{7}$ The service is provided by community pharmacies who buy the unit-packed pouches primarily from two nationally operating companies. ${ }^{1,2}$

Within the selected one-month period in September 2015, the structured register data for this study was derived from PharmaService's documentation system, ANJA. The patients' selection criteria was the following: age $\geq 65$ years, the first MDD order from PharmaService was made in September 2015, there were no missing data in MDD records and the service was used at least for the period of the subsequent 6 months.

The records contained anonymous structured information on patients' gender, age, underlying diseases, and MDD medications (name and dosage of medications with ATC-code and time of administration). The MDD-medication lists of the same patients were collected again after six months from the enrolment in the MDD service for the followup.

The appropriateness of the medications in terms of PIM use was evaluated manually by comparing the patient's medication list at baseline and at 6 months to the EU(7)-PIM list. ${ }^{8}$ If the potential harmfulness of the medication was dependent on the dose or duration of the treatment or medication itself, it was taken into account in the medication review. Identified PIMs were divided as clinically significant (used medications were inappropriate for older patients or it was used in higher doses and/or longer treatment course as recommended) and non-significant (medications were used in lower doses and/or shorter treatment course as considered harmful for older patients) PIMS. The EU(7)-PIM list was used as a reference because it is adopted to be used within EU, it is quite recently developed (in 2014) and it can be used if the available clinical information is minimal. ${ }^{8}$

The clinically significant drug-drug interactions were identified also manually by using Inxbase system. ${ }^{9}$ It was selected as a criteria because it is routinely used in Finland throughout the healthcare, also in community pharmacies. ${ }^{10}$ The Inxbase suggests short, concise and evidencebased information on more than 20,000 drug interactions that are divided into four levels (A-D) of clinical relevance, where A means minor interaction and D means interaction that is best avoided. ${ }^{9}$ In this study, category $\mathrm{C}$ and $\mathrm{D}$ interactions were documented, with the main focus on type D clinically significant DDIs.

\section{Data analysis}

The structured patient data from PharmaService's documentation system were entered into MS Excel 2010. Information on PIM use and DDIs based on medication reviews was also entered into Excel sheet. A Pearson's chi-squared test was used to assess the influence of background variables (age, gender, MDD medications, and the number of underlying chronic diseases per patient) on the number of PIMs and clinically relevant interactions.

\section{Results}

\section{Study participants}

Of the original sample of 622 patients initiating the MDD service, 208 (33\%) fulfilled the inclusion criteria and were included in the study (Fig. 1). Of the final study participants $(n=208), 69 \%$ were female (Table 1). The mean age of the participants was 84.6 years (range 65-108 years). The patients had on average 2.1 chronic conditions of which cardiovascular diseases (89\% of the participants), Alzheimer's disease (33\%) and severe mental disorders (26\%) were most common.
The mean number of prescribed MDD medications and food supplements taken were 5.9 and 1.3, respectively. The most frequently used groups of medications in treatment schemes were psychoanaleptics (85\%), beta-blockers (64\%), and dietary supplements (59\%). During the 6-month follow-up period, the number of medications used increased for $61 \%$ of the patients, and in most of the cases at least two medications and one food supplement had been added to the drug therapy regimen.

\section{Identification of PIMs}

At baseline, a majority ( $\mathrm{n}=152 ; 73 \%$ ) of the MDD patients used at least one PIM medication (Fig. 2). For $77 \%$ of the study participants $(\mathrm{n}=160)$, at least one medication from the EU(7)-PIM list was prescribed after the 6-months' period, and about $62 \%((\mathrm{n}=122(59 \%)$ for the first month, and $n=134$ (64\%) after 6 months of using MDD service)) of these PIMs were clinically significant. With the increase in the number of medications, the risk of PIMs in the patient's medication list increased ( $p<0.01$ ). PIM users were more likely to take five or more medications daily (Table 2). The most common potentially harmful PIMs were associated with acetylsalicylic acid, pantoprazole, carbamazepine and risperidone (Fig. 3).

\section{Prevalence of clinically significant DDIs}

Prevalence of clinically significant DDIs depended on the number of medications used $(p<0.01)$ and the number of diseases $(p=0.01)$. Patients exposed to potential DDIs used five or more medications daily, and had four or more chronic diseases (Table 2). According to the Inxbase database, 104 patients (50\%) had category C and only five patients $(2.4 \%)$ had category D DDIs. In total, three D-interactions with carbamazepine (carbamazepine + nifedipine/quetiapine/risperidone), one D-interaction of citalopram and sulpiride, and one D-interaction of spironolactone and potassium were found. The mentioned category D interactions were identified both at the first and six months after initiating the MDD service.

\section{Discussion}

To the authors' best knowledge, this is the first study evaluating drug related problems based on the EU(7)-PIM list and Inxbase databases of older patients using MDD service. Both above listed tools complemented each other and could be recommended for evaluation of medication use of older patients in other European countries. In the present study, there was no control group used. However, a cross-sectional study with the control group (e.g., MDD with and without medication review included in the service) could be developed to identify more specific outcomes and to determine the way forward in regard to the patients' safety when using MDD service.

Wallerstedt et al. (2013) ${ }^{4}$ and Sjöberg et al. $(2011)^{6}$ reported that the MDD system increases the number of medications and the findings could not be explained by the increased disease burden for patients with MDD. ${ }^{4,6}$ The current study supports results of previous research. However, the mechanism of the increase in the number of medications during the MDD service remains unclear and needs further research. The following reasons could be considered for the increased number of used medications. Firstly, the temporal association between the MDD and an increased proportion of used medications indicates that the drug treatment may be more seldom reconsidered and new medications added. Secondly, the increased adherence to the medications may manifest in new DRPs such as adverse reactions that need to be treated. Underlying mechanisms and contributing factors for our findings can only be speculated upon, but need further research.

Prevalence of DDIs and PIMs varies considerably in the literature and depending on the differences in patient and disease characteristics, prescribing patterns, and the availability of medications listed in DDI or 


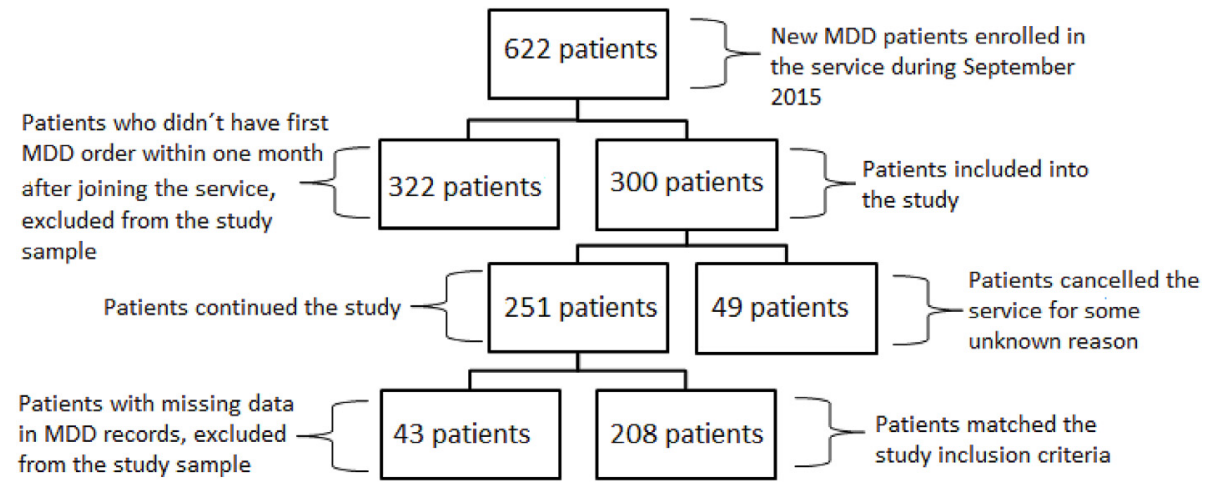

Fig. 1. Selection of the patients for the study $(n=208)$.

Table 1

Characteristics of the multi-dose dispensing (MDD) patients $(n=208)$ included in the study.

\begin{tabular}{|c|c|c|}
\hline Variable & $\mathrm{n}$ & $\%$ \\
\hline \multicolumn{3}{|l|}{ Gender } \\
\hline Female & 143 & 68.8 \\
\hline Male & 65 & 31.2 \\
\hline \multicolumn{3}{|l|}{ Age (years) } \\
\hline $65-90$ & 174 & 83.7 \\
\hline$>90$ & 34 & 16.3 \\
\hline \multicolumn{3}{|l|}{ Chronic diseases } \\
\hline Mean number of chronic diseases per person & 2.1 & \\
\hline Cardiovascular diseases (at least one condition); e.g., hypertension & 186 & 89.0 \\
\hline Alzheimer's dementia & 69 & 33.2 \\
\hline $\begin{array}{l}\text { Severe psychotic and/or other severe mental disorders (at least one } \\
\text { condition) }\end{array}$ & 55 & 26.4 \\
\hline Type II diabetes & 30 & 25.0 \\
\hline $\begin{array}{l}\text { Asthma and similar obstructive pulmonary diseases (at least one } \\
\text { condition) }\end{array}$ & 19 & 14.4 \\
\hline \multicolumn{3}{|l|}{ MDD packed medications and food supplements } \\
\hline $\begin{array}{l}\text { Mean number of medications per person (prescription and non- } \\
\text { prescription medicines) }\end{array}$ & 5.9 & \\
\hline Mean number of food supplements per person & 1.3 & \\
\hline Psychoanaleptics (at least one in use); e.g., amitriptyline & 176 & 84.6 \\
\hline Beta blockers (at least one in use); e.g., metoprolol & 132 & 63.5 \\
\hline Dietary supplements (at least one in use); e.g., calcium carbonate & 122 & 58.7 \\
\hline
\end{tabular}

PIM criteria. Some studies indicate that MDD users may be more exposed to PIM use but at the same time have lower probability of potentially dangerous DDIs. ${ }^{6,11,12}$ The present study showed that up to $64 \%$ of the MDD service patients have a risk of clinically significant PIMs, and $2.4 \%$ are susceptible to clinically significant category D drug interactions. The most common PIMs were associated with
Table 2

Dependence between the number of pouched medications used, the number of chronic diseases, the prevalence of potentially inappropriate medications (PIMs), and the prevalence of C and D class drug-drug interactions (DDIs) based on the Inxbase database among the multi-dose dispensing (MDD) patients within 6 months $(\mathrm{n}=208)$.

\begin{tabular}{|c|c|c|c|c|c|c|}
\hline & \multicolumn{2}{|c|}{$\begin{array}{l}\text { Patients with no } \\
\text { (clinically significant) } \\
\text { PIMs }(\mathrm{n}=76)\end{array}$} & \multicolumn{2}{|c|}{$\begin{array}{l}\text { Patients with clinically } \\
\text { significant PIMs } \\
(\mathbf{n}=132)\end{array}$} & \multicolumn{2}{|c|}{$\begin{array}{l}\text { All patients } \\
(\mathrm{n}=208)\end{array}$} \\
\hline & n & $\%$ & $\mathbf{n}$ & $\%$ & $\mathbf{n}$ & $\%$ \\
\hline \multicolumn{7}{|c|}{ Number of chronic diseases per person } \\
\hline $1-3$ & 68 & 37.4 & 114 & 62.6 & 182 & 87.5 \\
\hline $4-6$ & 8 & 30.8 & 18 & 69.2 & 26 & 12.5 \\
\hline \multicolumn{7}{|c|}{ Number of MDD medications used per person* } \\
\hline $1-5$ & 41 & 62.1 & 25 & 37.9 & 66 & 31.7 \\
\hline $5-9$ & 29 & 24.6 & 89 & 75.4 & 118 & 56.7 \\
\hline \multirow[t]{3}{*}{$9-13$} & 6 & 25 & 18 & 75 & 24 & 11.6 \\
\hline & \multicolumn{2}{|c|}{$\begin{array}{l}\text { Patients with no DDIs } \\
\qquad(\mathrm{n}=99)\end{array}$} & \multicolumn{2}{|c|}{$\begin{array}{l}\text { Patients with DDIs } \\
(\mathrm{n}=109)\end{array}$} & \multicolumn{2}{|c|}{$\begin{array}{l}\text { All patients } \\
(\mathrm{n}=208)\end{array}$} \\
\hline & $\mathrm{n}$ & $\%$ & $\mathbf{n}$ & $\%$ & $\mathbf{n}$ & $\%$ \\
\hline \multicolumn{7}{|c|}{ Number of chronic diseases per person* } \\
\hline $1-3$ & 93 & 51.1 & 89 & 48.9 & 182 & 87.5 \\
\hline $4-6$ & 6 & 23.1 & 20 & 76.9 & 26 & 12.5 \\
\hline \multicolumn{7}{|c|}{ Number of medications used per person* } \\
\hline $1-5$ & 46 & 69.7 & 20 & 30.3 & 66 & 31.7 \\
\hline $5-9$ & 47 & 39.8 & 71 & 60.2 & 118 & 56.7 \\
\hline $9-13$ & 6 & 25 & 18 & 75 & 24 & 11.6 \\
\hline
\end{tabular}

*p $<0.001$.

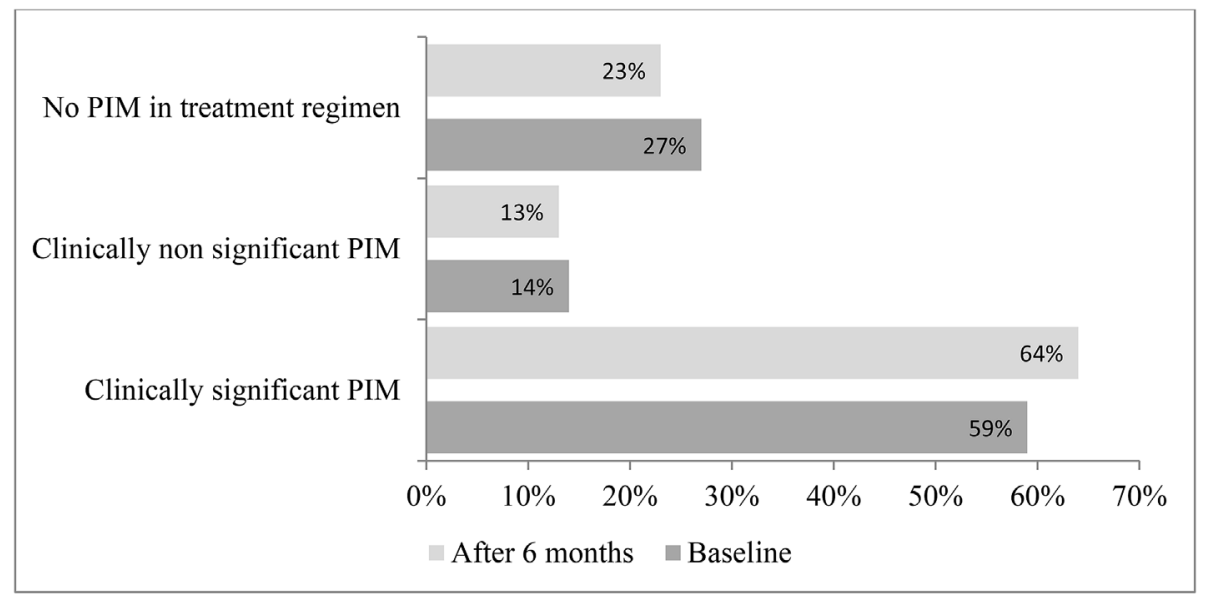

Fig. 2. Patients with PIMs and with no PIMs in the treatment regimen at baseline and after 6 months from entering the MDD service (\% of the patients, $\mathrm{n}=208)$. 


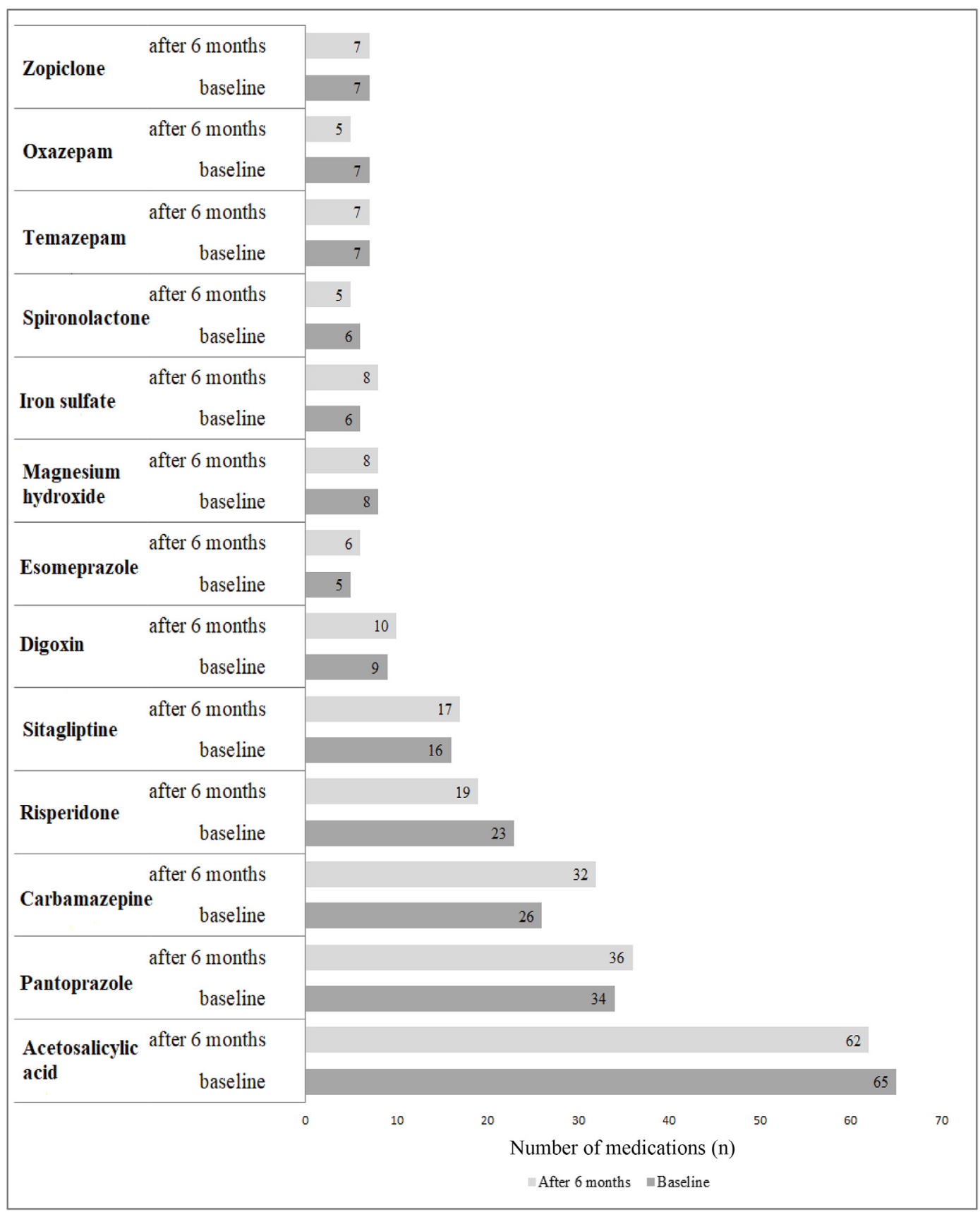

Fig. 3. The incidence of the most common potentially inappropriate medications (PIMs) (n) identified among the patients $(\mathrm{n}=208)$ during a 6-month follow-up period in the multi-dose drug dispensing (MDD) service.

acetylsalicylic acid with the risk of GI ulcers and bleedings, and clinically significant PIMs with pantoprazole taken for a longer period than recommended with an increased risk of Clostridium difficile infection and hip fracture. These findings are consistent with those reported by Hosia-Randell et al. (2008). ${ }^{12}$ To decrease number of PIMs comprehensive medication review should be properly conducted before entering patients in and regularly during the MDD service. ${ }^{1,2}$ There could be developed international guidelines for scheduled medication reviews of MDD patients where pharmacists could more closely collaborate with physicians. For more effective evaluation of patients' medication record, joint regular CPD courses could be developed for different healthcare specialists.

\section{Study limitations}

The study excludes data on patients with prescription and nonprescription medications not included in the MDD service. This may lead to an underestimation of medication use. The study identified only potential DRPs, and consequently, could not conclude how many of them can cause a manifest DRP, or how many would lead to a change being made in the drug treatment.

\section{Conclusions}

In this study, an MDD service was observed to have an association with greater frequency of PIM use among older patients in initiation common PIM - can become even common within 6 months. Despite the fact that all patients enrolled in the MDD service are recommended to 
receive a medication review service, a large proportion of the MDD patients used at least one PIM. Clinically significant DDIs were relatively rare but still of considerable concern. The results suggest that in the future, MDD services should be accompanied by a regular medication review tailored to specific patient groups (i.e., older patients) to avoid potential DRPs.

\section{Ethics approval}

This retrospective register-based study did not require ethical approval, since the study did not involve a prospective evaluation, and any laboratory animals or any clinical interventions to patients.

\section{Funding}

This research did not receive any specific grant from funding agencies in the public, commercial, or not-for-profit sectors.

\section{Conflicts of interest}

The authors declare that they have no conflict of interest.

\section{Acknowledgments}

The authors thank the following partners for their contribution: PharmaService Ltd., Finland.

\section{References}

1. Sinnemäki J, Saastamoinen LK, Hannula S, Peura S, Airaksinen M. Starting an automated dose dispensing service provided by community pharmacies in Finland. Int J Clin Pharm. 2013;36:345-351.

2. Sinnemäki J, Airaksinen M, Valaste M, Saastamoinen LK. Impact of the automated dose dispensing with medication review on geriatric primary care patients drug use in Finland: a nationwide cohort study with matched controls. Scand J Prim Health Care. 2017;35(4):379-386.

3. Reuther L, Lysen C, Faxholm M, Salomon L, Hendriksen C. Multi-dose drug dispensing is a challenge across the primary-secondary care interface. Dan Med Bull. 2011;58(12):A4341.

4. Wallerstedt SM, Fastbom J, Johnell K, Sjöberg C, Landahl S, Sundström A. Drug treatment in older people before and after the transition to a multi-dose drug dispensing system - a longitudinal analysis. PloS One. 2013;8(6):1-8 e67088.

5. Hammar T, Hovstadius B, Lidström B, Petersson G, Eiermann B. Potential drug related problems detected by electronic expert support system in patients with multidose drug dispensing. Int J Clin Pharm. 2014;36:943-952.

6. Sjöberg C, Edward C, Fastbom J, et al. Association between multi-dose drug dispensing and quality of drug treatment - a register-based study. PloS One. 2011;6(10):1-5 e26574.

7. The Association of Finnish Pharmacies - Annual Review. Medicines in pre-packed doses. http://www.apteekkariliitto.fi/media/pdf/annual_report_2003.pdf 2003 Accessed 10.10.2018

8. Renom-Guiteras A, Meyer G, Thürmann PA. The EU(7)-PIMlist: a list of potentially inappropriate medications for older people consented by experts from seven European countries. Eur J Clin Pharmacol. 2015;71:861-875.

9. Inxbase. https://ravimid.med24.ee/Accessed 15.11.2017.

10. Toivo TM, Mikkola JA, Laine K, Airaksinen M. Identifying high risk medications causing potential drug-drug interactions in outpatients: a prescription database study based on an online surveillance system. Res Soc Adm Pharm. 2016 JulAug;12(4):559-568.

11. Johnell K, Fastbom J. Multi-dose drug dispensing and inappropriate drug use: nationwide register-based study of over 700,000 elderly. Scand J Prim Health Care. 2008;26:86-91.

12. Hosia-Randell HMV, Muurinen SM, Pitkälä KH. Exposure to potentially inappropriate drugs and drug-drug interactions in elderly nursing home residents in Helsinki, Finland - a cross-sectional study. Drugs Aging. 2008;25(8):683-692. 